\section{Gustavo A. Obando' Karla E. Torres ${ }^{2}$}

Especialista en Implantología Especialista en Odontopediatría.

Facultad de Odontología de Piracicaba. Universidad Estadual de Campinas - UNICAMP. Brasil.

\section{Correspondencia}

Gustavo Obando Pereda

Avenida Limeira 901, Bairro Areiao, CEP 13414-

903, Piracicaba, Sao Paulo, Brasil.

Telefono: 0055-19-21065321

e-mail: gustavo_obandop@yahoo.com.ar

Recibido : 15 de enero del 2007

Aceptado : 15 de junio del 2007

\title{
Efecto del triclosán sobre el biofilm del cepillo dental
}

Obando GA, Torres KE. Efecto del triclosán sobre el biofilm del cepillo dental. Rev Estomatol Herediana. 2007; 17(1):25-28.

\section{RESUMEN}

Se evaluó el efecto antimicrobiano de dos dentífricos sobre la flora aeróbica del cepillo dental en uso. El estudio se realizo con treinta individuos entre los 20 y 30 años de edad que se cepillaron los dientes con un cepillo estándar. Quince usaron un dentífrico con triclosán y los restantes un dentífrico sin triclosán. Después de tres semanas se procesaron los cepillos y se obtuvo como resultado que el dentífrico con triclosán posee mayor efecto antimicrobiano sobre el biofilm del cepillo dental que el dentífrico sin triclosán.

\section{Palabras clave: DISPOSITIVOS PARA EL AUTOCUIDADO BUCAL / DENTÍFRICOS} BIOFILMES / TRICLOSÁN.

\section{Effect of triclosán dentifrice on biofilm from toothbrush}

ABSTRACT

The present study assessed the antimicrobial effect of two commercial dentifrices against aerobic microflora in used standard toothbrushes. The study was carried out on 30 healthy people ranging between 20 and 30 years of age. Fifteen used a dentifrice without triclosan, and the other fifteen a dentifrice containing triclosan. After 3 weeks the toothbrushes were microbiologically processed. The results of this study demonstrated that the dentifrice with triclosan has greater effect against toothbrush biofilm than the dentifrice without triclosan.

Key words: HOME CARE DENTAL DEVICES / DENTIFRICES / BIOFILMS / TRICLOSÁN.

\section{Introducción}

Existe evidencia científica de que la transmisión de microorganismos de la boca puede ocurrir a través de la saliva (1). La translocación de especies cariogénicas y periodontopatógenas puede ser producida por el uso de instrumentos dentales así como instrumentos de higiene oral (2-5). La retención y sobrevivencia de microorganismos orales en el cepillo dental representa una posible fuente de recontaminación de la boca, convirtiéndolo en potencial re introductor de patógenos a la cavidad oral (68). El uso prolongado del cepillo, el incremento de microorganismos en el cepillo, y una recontaminación oral con microorganismos puede causar infecciones orales como la caries, gingivitis y estomatitis (8).

Estos microorganismos pueden transferirse de diversas fuentes tales como del aerosol emanado del inodoro, de los dedos de la persona, de los sitios húmedos del cuarto de baño y en general del medio ambiente $(6,8)$.

Glass y Lare (6) sugirieron que la contaminación del cepillo dental juega un rol importante como puerta de entrada de una enfermedad sistémica, ya que debido al uso de una inapropiada técnica de cepillado, el usuario puede lacerar la encía favoreciendo la introducción de microorganismos al torrente sanguíneo provenientes de la flora presente en el cepillo.

El dentífrico y la saliva proporciona condiciones favorables para la manutención de la microflora en el cepillo dental, la composición del dentífrico es un factor para que combatir esta microflora $(9,10)$. Recientemente se ha introducido dentífricos que contienen triclosán en su composición el cual es un compuesto utilizado comúnmente para desinfección (11).

En el presente trabajo de investigación se evaluó el efecto antimicrobiano del triclosán, sobre el biofilm residual del cepillo dental que se forma después de su uso.

\section{Material y métodos}

El estudio se llevo a cabo en 30 individuos adultos que cumplían con los siguientes requisitos de inclusión (11):

- Ausencia de bolsas periodontales mayores a $3 \mathrm{~mm}$.

- Mujeres no embarazadas y que no hayan usado terapia hormonal en los seis meses previos al estudio

- No uso de medicamentos en los seis meses previos al estudio

- No padecer de condiciones sistémicas graves o de consideración que pueda afectar la salud bucal.

- No hayan usado dentífricos con triclosán.

- Poseer como mínimo tres dientes posteriores en cada cuadrante.

El rango de edad de los sujetos estuvo entre 20 y 30 años. Todos usaron un cepillo dental estándar, 15 de ellos se cepillaron los dientes con un dentífrico sin triclosán (Colgate Antisarro $\left.{ }^{\circledR}\right)$ y a los restantes, con un dentífrico con triclosán (Colgate Total $\left.{ }^{\circledR}\right)$. Se les indicó a los usuarios que sigan sus hábitos de higiene normales por tres semanas, recolectándose luego los cepillos en una bolsa de papel estéril, procediéndose luego al estudio microbiológico (7). 
El procesamiento microbiológico se realizó a las 18 horas después de obtenidos los cepillos. Una vez obtenidos, se les separó la cabeza (parte del cuello y las cerdas) y se las colocó, individualmente en un frasco estéril con $10 \mathrm{ml}$ de suero fisiológico. Este contenido, es vigorosamente mezclado por 90 segundos para luego hacer la dilución respectiva, procediendo al repique de $1 \mathrm{ml}$ del inóculo en agar sangre, agar Mc Conkey y agar Chapman. Se deja cultivar por 24 horas a $37^{\circ} \mathrm{C}$, para luego proceder al conteo de colonias y su diferenciación microbiológica (7).

Para verificar la existencia de diferencia en el efecto antimicrobiano de los dos dentífricos, se utilizaron dos pruebas estadísticas: la $t$ de Student, para comparar medias aritméticas y la prueba de Kruskal Wallis, para comparar varianzas $(p<0,05)$. Se usó el software SPSS $12.0 \mathrm{v}$.

\section{Resultados}

El Streptococcus spp fue el microorganismo más numeroso obte- nido de los cepillos que utilizaron dentífrico sin triclosán (Tabla 1).

En los cepillos que utilizaron dentífrico con triclosán se pudo identificar al Bacillus subtilis como el microorganismo más frecuente (Tabla 2)

Los cepillos en los que se uso dentífrico sin triclosán mostraron un mayor número de unidades formadoras de colonias (UFC) (Tabla 3).

La actividad antimicrobiana, medida como presencia de UFC fue menor estadísticamente significativa $(\mathrm{P}<0,001)$ en los cepillos donde se usaron dentífrico con triclosán (Fig. 1).

Los cepillos que usaron dentífrico con triclosán obtuvieron bajo Riesgo Microbiológico y los otros obtuvieron un Riesgo Microbiológico moderado (Fig. 2).

\section{Discusión}

Con el presente trabajo, se ha podido corroborar los resultados obtenidos en las investigaciones de Glass y Lare (6) y Taji y Rogers (7), en los cuales también se encontraron similares microorganismos.

El efecto antimicrobiano del dentífrico sin triclosán, es bajo debido a que en sus ingredientes no existen productos antimicrobianos, ya que sólo posee ingredientes aromatizantes, blanqueadores, detergentes y soluciones fluoradas.

El dentífrico con triclosán, además contiene otros ingredientes antimicrobianos como son el metilparabeno y propilparabeno, los cuales presentan gran eficacia contra microorganismos Gram positivos y algunos negativos, además de ingredientes aromatizantes, detergentes y soluciones fluoradas (11).

Los microorganismos encontrados en los cepillos dentales que usaron dentífrico sin triclosán corresponden a microorganismos autóctonos de la cavidad oral como también microorganismos procedentes del medio ambiente (alóctonos). Los microorganismos encontrados en los cepillos dentales que usaron dentífrico con triclosán pertenecen a microorganismos alóctonos (microorganismos transitorios), los cuales provienen del medio ambien-

Tabla 1. Unidades Formadoras de Colonias (UFC) $10^{2} / \mathrm{ml}$ en cepillo donde se uso dentífrico sin triclosán.

\begin{tabular}{lrrrrrrrrrrrrrrrrr}
\hline Elementos & $\mathrm{A}$ & $\mathrm{B}$ & $\mathrm{C}$ & $\mathrm{D}$ & $\mathrm{E}$ & $\mathrm{F}$ & $\mathrm{G}$ & $\mathrm{H}$ & $\mathrm{I}$ & $\mathrm{J}$ & $\mathrm{K}$ & $\mathrm{L}$ & $\mathrm{M}$ & $\mathrm{N}$ & $\tilde{\mathrm{N}}$ & $\mathrm{O}$ & $\mathrm{P}$ \\
\hline 1 & 0 & 0 & 0 & 0 & 0 & 0 & 0 & 0 & 47 & 0 & 62 & 0 & 0 & 0 & 0 & 0 & 0 \\
2 & 0 & 3 & 0 & 0 & 0 & 0 & 0 & 2 & 2 & 0 & 5 & 0 & 0 & 0 & 0 & 0 & 0 \\
3 & 1 & 17 & 0 & 0 & 0 & 0 & 0 & 5 & 12 & 1 & 0 & 2 & 0 & 0 & 0 & 0 & 0 \\
4 & 0 & 0 & 0 & 0 & 0 & 0 & 0 & 0 & 920 & 0 & 0 & 0 & 140 & 0 & 24 & 1 & 0 \\
5 & 11 & 58 & 0 & 0 & 0 & 0 & 0 & 1 & 19 & 0 & 0 & 0 & 0 & 0 & 0 & 0 & 0 \\
6 & 17 & 63 & 0 & 0 & 0 & 0 & 0 & 0 & 0 & 0 & 0 & 2 & 0 & 0 & 0 & 0 & 0 \\
7 & 0 & 1 & 2 & 0 & 0 & 0 & 0 & 1 & 9 & 0 & 0 & 0 & 0 & 6 & 0 & 0 & 1 \\
8 & 4 & 10 & 0 & 0 & 1 & 0 & 0 & 0 & 2 & 0 & 0 & 0 & 0 & 0 & 0 & 0 & 0 \\
9 & 5 & 0 & 2 & 0 & 0 & 0 & 0 & 0 & 0 & 1 & 0 & 2 & 0 & 0 & 0 & 0 & 0 \\
10 & 0 & 0 & 0 & 0 & 0 & 0 & 0 & 15 & 0 & 0 & 0 & 0 & 0 & 0 & 0 & 0 & 0 \\
11 & 0 & 0 & 0 & 0 & 0 & 0 & 1 & 1 & 0 & 0 & 0 & 0 & 0 & 0 & 0 & 0 & 0 \\
12 & 18 & 5 & 0 & 3 & 0 & 0 & 0 & 1 & 9 & 1 & 0 & 0 & 0 & 0 & 0 & 0 & 0 \\
13 & 1 & 2 & 0 & 0 & 0 & 0 & 0 & 1 & 0 & 0 & 0 & 2 & 0 & 0 & 0 & 0 & 0 \\
14 & 17 & 1 & 0 & 0 & 0 & 0 & 0 & 5 & 1012 & 24 & 0 & 0 & 0 & 0 & 0 & 140 & 0 \\
15 & 20 & 0 & 20 & 0 & 0 & 58 & 0 & 60 & 1268 & 36 & 0 & 0 & 0 & 0 & 0 & 0 & 68 \\
\hline
\end{tabular}

$\mathrm{A}=$ Staphylococus albus, $\mathrm{B}=$ Corynebacterium spp, $\mathrm{C}=$ Staphylococcus aureus, $\mathrm{D}=$ Moraxella catarralis, $\mathrm{E}=$ Pseudomonas aeruginosa, $\mathrm{F}=$ Streptococcus pneumoniae, $\mathrm{G}=$ Enterobacterium aerogenes, $\mathrm{H}=$ Bacillus subtilis, $\mathrm{I}=$ Streptococcus spp, $\mathrm{J}=\mathrm{E}$. coli, $\mathrm{K}=$ Micrococcus spp, L=Bacillus Gram negativo no fermentador, M=Difteroides spp, $\mathrm{N}=$ Candida albicans, $\tilde{\mathrm{N}}=$ Streptococcus $\beta$ hemolítico, $\mathrm{O}=$ Moraxella morganii, $\mathrm{P}=$ Enterococus cloacae. 
Tabla 2. Unidades Formadoras de Colonias (UFC) $10^{2} / \mathrm{ml}$ en cepillo donde se uso dentífrico con triclosán.

\begin{tabular}{llllllll}
\hline Elementos & $\mathrm{A}$ & $\mathrm{B}$ & $\mathrm{C}$ & $\mathrm{D}$ & $\mathrm{E}$ & $\mathrm{F}$ & $\mathrm{G}$ \\
\hline 1 & 2 & 0 & 0 & 0 & 0 & 0 & 0 \\
2 & 2 & 0 & 0 & 0 & 0 & 0 & 0 \\
3 & 1 & 0 & 0 & 0 & 0 & 0 & 0 \\
4 & 0 & 0 & 2 & 0 & 0 & 0 & 0 \\
5 & 0 & 0 & 1 & 0 & 0 & 0 & 0 \\
6 & 0 & 0 & 0 & 3 & 4 & 0 & 0 \\
7 & 0 & 0 & 0 & 0 & 0 & 1 & 0 \\
8 & 0 & 0 & 0 & 0 & 0 & 0 & 3 \\
9 & 0 & 0 & 0 & 0 & 6 & 0 & 0 \\
10 & 0 & 4 & 0 & 0 & 6 & 0 & 0 \\
11 & 0 & 0 & 0 & 0 & 5 & 0 & 0 \\
12 & 0 & 4 & 0 & 0 & 4 & 0 & 0 \\
13 & 0 & 0 & 0 & 3 & 0 & 0 & 0 \\
14 & 0 & 2 & 0 & 0 & 0 & 0 & 0 \\
15 & 0 & 0 & 0 & 0 & 4 & 0 & 0 \\
\hline $\mathrm{A}=\mathrm{E}$ & $\mathrm{B}$ & 0 & &
\end{tabular}

$\mathrm{A}=\mathrm{E}$. coli, $\mathrm{B}=$ Sthapylococcus saprofiticcus, $\mathrm{C}=$ Candida albicans, $\mathrm{D}=$ Staphylococcus aureus, $\mathrm{E}=$ Bacillus subtilis, $\mathrm{F}=$ Moraxella catarralis, $\mathrm{G}=$ Staphylococcus albus.

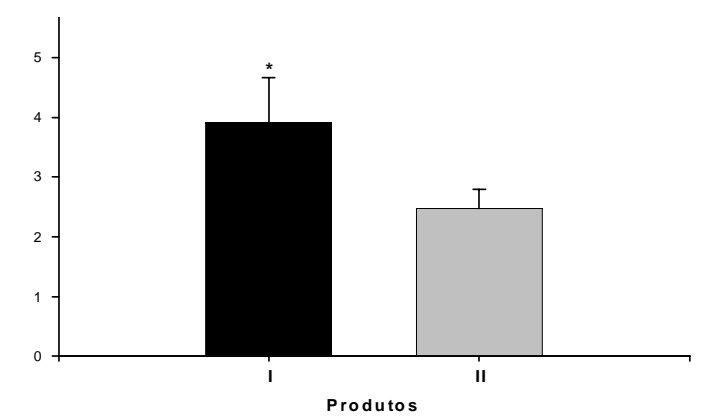

Fig. 1. Biofilm residual identificado en los cepillos dentales ( $\mathrm{I}=$ cepillo $\sin$ triclosán, $\mathrm{II}=$ cepillo con triclosán.
Tabla 3. Cantidad de Unidades Formadoras de Colonias ( $\left.\log _{10} \mathrm{UFC} / 10 \mathrm{ml}\right)$ según tipo de dentífrico usado.

\begin{tabular}{llll}
\hline Elementos & Sin triclosán & Elementos & Con triclosán \\
\hline 1 & 4,0374 & 1 & 2,3010 \\
2 & 3,0792 & 2 & 2,3010 \\
3 & 3,7076 & 3 & 2,0000 \\
4 & 5,0366 & 4 & 2,3010 \\
5 & 3,9494 & 5 & 2,0000 \\
6 & 4,1523 & 6 & 2,8451 \\
7 & 3,3010 & 7 & 2,0000 \\
8 & 2,9031 & 8 & 2,4771 \\
9 & 4,4502 & 9 & 2,7782 \\
10 & 3,1761 & 10 & 2,9542 \\
11 & 3,8388 & 11 & 2,6990 \\
12 & 3,5682 & 12 & 2,9031 \\
13 & 3,1761 & 13 & 2,4771 \\
14 & 5,0792 & 14 & 2,3010 \\
15 & 5,1761 & 15 & 2,6021 \\
\hline
\end{tabular}

te con excepción de la C. albicans y el Staphylococcus aureus.

Estos últimos microorganismos están relacionados exclusivamente con el cuidado que se da al cepillo dental post higiene oral. Se recomienda que el cepillo dental debe estar guardado en un lugar libre de contaminación, seco, aireado y alejado del inodoro en más de un metro y medio.

Debido a la gran existencia de microorganismos en los cepillos que usaron dentífrico sin triclosán, parece adecuado recomendar en la consulta particular y/o en la práctica pública la utilización de dentífricos que en su composición tengan triclosán. Rule, Ebbett y Vikesland (12), investigaron la formación de cloroformo y compuestos orgánicos clorados por oxidación del triclosán al contacto con el agua potable, ambos productos tienen gran potencial mutagénico, atribuyéndosele un alto poder cancerígeno. Este estudio preliminar ha puesto en alerta a las comunidad científica es mundiales sobre el manejo del triclosán, así como la inmediata respuesta de las empresas cuyos productos contienen triclosán en su composición las cuales defienden su uso debido a que la cantidad de triclosán no es significativa para un trastorno de esa importancia, además de que todavía no hay muchas evidencias de cánceres atribuidos al cloroformo en humanos.

Glass y Lare (6) recomiendan que el cepillo dental debe ser desinfectado cada dos semanas con hipoclorito de sodio al 1\% ó gluconato de clorhexidina al $0,12 \%$ por cinco minutos, de lo contrario debe ser renovado.

El índice utilizado para medir el Riesgo Microbiológico es un índice modificado, cuyo original fue propuesto por Westergreen y Krasse (13), para medir el riesgo microbiológico del Streptococcus mutans y por el Lactobacillus spp. por $\mathrm{ml}$ de saliva. 


\section{Conclusiones}

El dentífrico con triclosán demostró poseer un mayor efecto antimicrobiano que el dentífrico sin triclosán.

Los cepillos dentales que se usaron con dentífrico con triclosán poseen un bajo riesgo microbiológico.

Los cepillos dentales que se usaron con dentífrico sin triclosán, en un tiempo de tres semanas, se convierten en instrumentos potenciales de reintroducción de microorganismos a la cavidad oral.

\section{Agradecimiento}

Facultad de Odontología de la Universidad Católica de Santa María. Facultad de Odontología de Piracicaba - UNICAMP.

\section{Referencias bibliográficas}

1. Van Winkelhoff AJ, Van der Velden U, Clement M, De Graaff J. Intra-oral distribution of blackpigmented Bacteroides species in periodontitis patients. Oral Microbiol Immunol. 1988; 3(2):83-85.

2. Edman DC, Keene HJ, Shklair IL, Hoerman KC. Dental floss for implantation and sampling of Streptococcus mutans from approximal surfaces of human teeth. Arch Oral Biol. 1975;
20(2):145-8.

3. Efstratiou M, Papaioannou W, Nakou M, Ktenas E, Vrotsos IA, Panis V. Contamination of a toothbrush with antibacterial properties by oral microorganisms. J Dent. 2007; 35(4):331-7.

4. Loesche WJ, Svanberg ML, Pape HR. Intraoral transmission of Streptococcus mutans by a dental explorer. J Dent Res. 1979; 58(8):1765-70.

5. Loesche WJ, Walenga A, Loos P. Recovery of Streptococcus mutans and Streptococcus sanguis from a dental explorer after clinical examination of single human teeth. Arch Oral Biol. 1973; 18(4):571-5.

6. Glass RT, Lare MM. Toothbrush contamination: a potential health risk? Quintessence Int. 1986; 17(1):39-42.

7. Taji SS, Rogers AH. ADRF Trebitsch Scholarship. The microbial contamination of toothbrushes. A pilot study. Aust Dent J. 1998; 43(2):128-30.

8. Wetzel WE, Schaumburg C, Ansari F, Kroeger T, Sziegoleit A. Microbial contamination of toothbrushes with different principles of filament anchoring. J Am Dent Assoc. 2005;
136(6):758-65.

9. Nelson-Filho P, Isper AR, Assed S, Faria G, Ito IY. Effect of triclosán dentifrice on toothbrush contamination. Pediatr Dent. 2004; 26(1):11-6.

10. Quirynen M, De Soete M, Pauwels M, Gizani S, Van Meerbeek B, van Steenberghe D. Can toothpaste or a toothbrush with antibacterial tufts prevent toothbrush contamination? J Periodontol. 2003; 74(3):312-22.

11. Warren DP, Goldschmidt MC, Thompson MB, Adler-Storthz K, Keene HJ. The effects of toothpastes on the residual microbial contamination of toothbrushes. J Am Dent Assoc. 2001; 132(9):1241-5.

12. Rule KL, Ebbett VR, Vikesland PJ. Formation of chloroform and chlorinated organics by freechlorine-mediated oxidation of triclosán. Environ Sci Technol. 2005; 39(9):3176-85.

13. Westergren G, Krasse B. Evaluation of a micromethod for determination of Streptococcus mutans and Lactobacillus infection. J Clin Microbiol. 1978; 7(1):82-3. 